\title{
Metabolic Effects of L-carnitine on Type 2 Diabetes Mellitus: Systematic Review and Meta-analysis
}

Authors

Affiliations
A. Vidal-Casariego ${ }^{1}$, R. Burgos-Peláez ${ }^{2}$, C. Martínez-Faedo ${ }^{3}$, F. Calvo-Gracia ${ }^{4}$, M. Á. Valero-Zanuy ${ }^{5}$, L. M. Luengo-Pérez ${ }^{6}$, C. Cuerda-Compés

Affiliation addresses are listed at the end of the article

\author{
Key words \\ total cholesterol \\ - low density lipoprotein \\ - tryglicerides \\ - lipoprotein(a) \\ - apolipoprotein-B100 \\ - apolipoprotein-A1 \\ 0 oxidative stress
}

received 26.11.2012 first decision 20.12.2012 accepted $\quad 10.01 .2013$

\section{Bibliography}

DOI http://dx.doi.org/

10.1055/s-0033-1333688

Published online:

February 21, 2013

Exp Clin Endocrinol Diabetes

2013; 121: 234-238

(c) J. A. Barth Verlag in Georg Thieme Verlag KG

Stuttgart · New York

ISSN 0947-7349

\section{Correspondence}

\section{A. V. Casariego}

Sección de Endocrinología

y Nutrición

Complejo Asistencial

Universitario de León

Altos de Nava SN

24008 León

Spain

Tel.: + 34/98/72374993044

Fax: + 34/98/724 0503

avcyo@hotmail.com

\section{Abstract}

$\nabla$

Introduction: Carnitine is an endogenous metabolite and exogenous nutrient with a pivotal role in lipid metabolism. Plasma levels of carnitine are reduced in type 2 Diabetes Mellitus (T2DM). The aim was to evaluate the metabolic effects of the administration of L-carnitine in T2DM.

Method: A systematic review was performed. Relevant randomized, controlled-trials trials were searched in Pubmed, Trip Database and Cochrane Library, and selected when they had enough methodological quality assessed with the Jadad scale. Article search strategy included "Carnitine" OR "L-carnitine" AND "Diabetes Mellitus" OR "Diabetes mellitus, type 2" OR "Noninsulindependent-diabetes mellitus". Metaanalysis was performed, and the difference of

\section{Introduction}

$\nabla$

L-carnitine is a non-protein amino acid which main sources in humans include both diet and endogenous synthesis. It is widely found in animal foods such as meat, fish, milk and dairy products, and it can be synthesised in liver and kidney from the amino acids lysine and methionine. Carnitine plays a role in lipid metabolism by regulating fatty acid (FA) transport between the cytosol and mitochondria [1]. It also acts as a cofactor in beta-oxidation by facilitating long chain FA entrance into mitochondria in the form of acylcarnitine esters (carnitine-palmitoyl transferase system CPT-I and II) and the exit of acetyl groups from mitochondria to the cytosol (carnitine acyl transferase system).

A reduction in FA transportation to mitochondria facilitates triglyceride accumulation in the cytosol, which is related with the pathogenesis of insulin resistance (IR). Although the mechanisms means calculated with a $95 \%$ confidence interval. Heterogeneity was evaluated with the Q statistic. Results: The systematic review included 4 trials with 284 patients. Oral L-carnitine lowered fasting plasma glucose $[-14.3 \mathrm{mg} / \mathrm{dl}$ (CI95\% -23.2 to -5.4$) ; \mathrm{p}=0,002]$, total cholesterol [ $-7.8 \mathrm{mg} / \mathrm{dL}$ (95\%CI -15.5 to -0.1$) ; \mathrm{p}=0.09]$, low density lipoprotein $[-8.8 \mathrm{mg} / \mathrm{dl}$ (CI95\% -12.2 to -8.5), $\quad \mathrm{p}<0.0001], \quad$ apolipoprotein-B100 [ $-7.6 \mathrm{mg} / \mathrm{dl}$ (CI95\% - 13.6 to -1.6$) ; \mathrm{p}=0.013$ ] and apolipoprotein-A1 [ $-6.0 \mathrm{mg} / \mathrm{dl}$ (CI95\% - 10.5 $\mathrm{a}-1.5) ; \mathrm{p}=0.523$ ]. There was no significant heterogeneity. The changes in triglycerides, lipoprotein (a) or $\mathrm{HbA}_{1 \mathrm{c}}$ were not significant.

Conclusion: The administration of L-carnitine in type 2 diabetes mellitus is associated with an improvement in glycaemia and plasma lipids. by long chain acyl-CoA, c) non-metabolised FA accumulation in mitochondria, which could cause mitochondrial stress and IR [2]. These mechanisms may explain why carnitine, being a conditionally essential nutrient involved in bidirectional transportation of acyl-CoA in mitochondria, could reduce lipid overload and improve insulin-sensitivity.

Carnitine supplementation has been effective for reducing IR in fat-rich diet-fed mice without changing intake or weight [2]. In obese and diabetic mice, carnitine improved insulin-mediated glucose metabolism; furthermore, the circulating levels of acylcarnitine and its urinary excretion were increased [2]. In studies with healthy humans, carnitine infusions improved glucose 
metabolism measured with hyperinsulinaemic euglycaemic clamp, mainly by a non-oxidative mechanism that results in the accumulation of glycogen [3-5]. In type 2 Diabetes Mellitus (T2DM), carnitine infusion improved glucose oxidation and glycogen storage $[6,7]$. Among diabetic patients with associated complications, L-carnitine plasma levels are $25 \%$ lower than among those without complications, and it has been suggested that carnitine could be a useful treatment in T2DM $[8,9]$.

A systematic review and meta-analysis was performed to test the current evidence about the efficacy of L-carnitine supplementation in the amelioration of metabolic disturbances of T2DM patients.

\section{Methods}

$\nabla$

\section{Search strategy and inclusion criteria}

A bibliographic search for randomised, controlled trials testing the effects of L-carnitine against a placebo in T2DM was performed in Medline (PubMed), Trip Database, and Central (Cochrane Library) databases. Article search strategy included "Carnitine" OR "L-carnitine" AND “Diabetes Mellitus" OR “Diabetes mellitus, type 2" OR "Noninsulindependent-diabetes mellitus". The initial eligibility criteria for including studies in the review were the type of assay (randomised, double-blinded, controlled studies), species (humans), and language (English, Spanish). The identified studies were evaluated for inclusion by the reviewers, who were not blinded to authors, institutions or journal at any during the selection process. When several papers from a same study were found, the publication with higher methodological quality was selected. The adequacy of the selected articles was measured using the Jadad scale, which considers if the study is randomised (and the method of randomisation), double blinded (and the method of blindness) and the description of lost patients. A minimal punctuation of 3 was necessary for the inclusion in this review. The quality assessment of each article was independently performed by 2 different reviewers.

\section{Outcome measures}

The investigators independently extracted the following data, using a common form for this purpose: a) Glucose metabolism: fasting plasma glucose (FPG), postprandial glucose (PPG), $\mathrm{HbA}_{1 c}$ and insulin levels. b) Lipid metabolism: total cholesterol (TC), HDL-cholesterol, LDL-cholesterol, triglycerides, apolipoproteinA1, apolipoprotein-B100 and lipoprotein (a). c) Body weight and body mass index (BMI). d) Systolic and diastolic blood pressure (BP). e) Oxidative stress (OS) markers.

\section{Statistical analysis}

Mean and standard deviation of each quantitative variable were obtained. Meta-analysis was conducted after the aggregation of data using the inverse variance-fixed effect model, which accurately calculates global results when heterogeneity is assumed not to be present. The mean differences (MD) were calculated for each metabolic parameter, with their confidence intervals of 95\% (CI95\%). Heterogeneity, which represents the differences among studies that may prevent the aggregation of results, was evaluated with the $\mathrm{Q}$ test. This is a commonly used and simple test included in the random effects method that measures the differences among the individual result of each study and the average result It was considered that there was heterogeneity when $\mathrm{p}<0.05$, and that there were significant differences between interventions when CI95\% did not include the value 0 .

\section{Results}

$\nabla$

After the progressive selection process, 4 studies with a total of 284 patients were included in the review [10-13] ( $\bullet$ Fig. 1). These studies are summarised in $\odot$ Table 1 . In all of them, L-carnitine was administered orally in the intervention group.

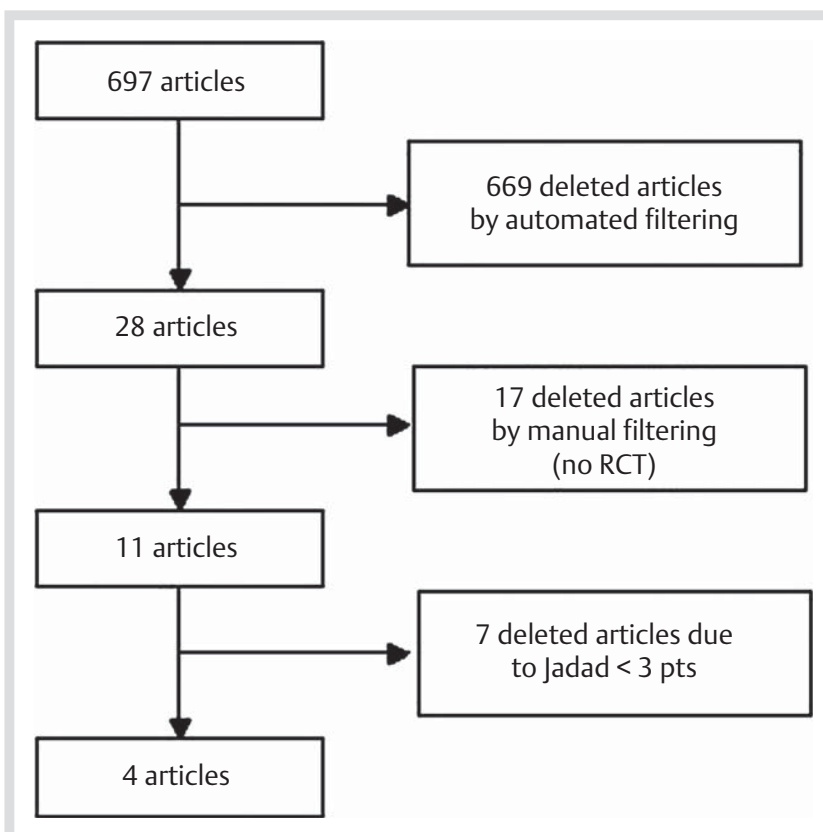

Fig. 1 Flow chart of the study selection process. RCT: Randomized, controlled trials.

Table 1 Summary of the selected articles.

\begin{tabular}{|c|c|c|c|c|c|c|c|}
\hline Author & Reference & Design & $\mathbf{N}$ & Intervention & $\begin{array}{l}\text { Duration } \\
\text { (weeks) }\end{array}$ & $\begin{array}{l}\text { Jadad } \\
\text { (points) }\end{array}$ & Outcomes \\
\hline Derosa & 9 & RCT & 94 & L-carnitine $2 \mathrm{~g} /$ day vs. placebo & 24 & 4 & Reduction of Lp(a). \\
\hline Rahbar & 10 & RCT & 35 & L-carnitine $3 \mathrm{~g} /$ day vs. placebo & 12 & 4 & $\begin{array}{l}\text { Reduction of FPG. Increases of } \mathrm{Tg} \text {, } \\
\text { apoA1 and apoB100. }\end{array}$ \\
\hline Santo & 11 & RCT & 74 & L-carnitine $2 \mathrm{~g} /$ day vs. placebo & 52 & 3 & Improvemento of ABI and FPG. \\
\hline Malaguarnera & 12 & RCT & 81 & L-carnitine $2 \mathrm{~g} /$ day vs. placebo & 12 & 4 & $\begin{array}{l}\text { Reduction in HDL, LDL, Tg, apoA1, } \\
\text { apoB100, and OS markers. }\end{array}$ \\
\hline
\end{tabular}

FPG: fasting glucose. HDL: high density lipoprotein. LDL: low density lipoprotein. Tg: triglycerides. apoA1: apoprotein A1. apo B100: apoprotein B100. Lp(a): lipoprotein a. ABI: ankle/branchial index. OS: oxidative stress 


\section{Effects on glucose metabolism}

The changes in FPG were evaluated in the 4 studies ( 0 Fig. 2 ). When the meta-analysis was performed, a MD of $-14.3 \mathrm{mg} / \mathrm{dL}$ (95\%CI -23.2 to $-5.4 ; \mathrm{p}=0,002)$ favoured the group treated with L-carnitine, without evidence of heterogeneity $\left(X^{2}=3.19\right.$, $\mathrm{p}=0.363)$. $\mathrm{HbA}_{1 \mathrm{c}}$ was evaluated in 3 of the included studies, and no significant effect was found after aggregating the results: $\mathrm{MD}=0.1 \%(95 \% \mathrm{CI}-0.7$ to $0.9 ; \mathrm{p}=0.892)$, without associated heterogeneity $\left(X^{2}=1.58 ; p=0.454\right)$. The study by Derosa et al. did not obtain significant differences between the L-carnitine and placebo groups regarding postprandial glycaemia and insulinaemia [9].

\section{Effects on lipid metabolism}

All studies included data about total cholesterol, HDL, LDL and triglycerides. The analysis of aggregated data showed a significant reduction in total cholesterol $[\mathrm{MD}=-7.8 \mathrm{mg} / \mathrm{dL}$ (95\%CI -15.5 to -0.1$) ; \mathrm{p}=0.09$; without heterogeneity: $\mathrm{X}^{2}=3.25$, $\mathrm{p}=0.280)]$ and LDL [MD $=-8.8 \mathrm{mg} / \mathrm{dL}(95 \% \mathrm{CI}-12.2$ to -8.5$)$; $\mathrm{p}<0.0001$; heterogeneity: $\mathrm{X}^{2}=2.78, \mathrm{p}=0.425$ ] associated with the administration of L-carnitine ( $\bullet$ Fig. 3,4 ). There was no significant changes in the concentration of HDL $[\mathrm{MD}=1.9 \mathrm{mg} / \mathrm{dL}$ $(95 \% \mathrm{CI}-1.2$ to 5.1$) ; \mathrm{p}=0.212$; without heterogeneity: $\mathrm{X}^{2}=3.41$, $\mathrm{p}=0.332$ ]. There was also no significant effect on triglycerides with the pooling of the 4 studies [MD $=-2.5 \mathrm{mg} / \mathrm{dL}(95 \% \mathrm{CI}-12.6$ to 7.6 ); $p=0.622$; without associated heterogeneity: $x^{2}=4.77$, $\mathrm{p}=0.190]$.

The effect of treatment on apolipoprotein AI was evaluated in 3 studies, and on apolipoprotein B100 and lipoprotein(a) in 2 studies. The use of L-carnitine was associated with a significant lowering of apolipoprotein AI $[\mathrm{MD}=-6.0 \mathrm{mg} / \mathrm{dL}(95 \% \mathrm{CI}-10.5$ to -1.5$) ; \mathrm{p}=0.008]$ and $\mathrm{B}-100[\mathrm{MD}=-7.6 \mathrm{mg} / \mathrm{dL}(95 \% \mathrm{CI}-13.6$ to -1.6$) ; \mathrm{p}=0.013$. There was no significant changes in the concentration of lipoprotein(a) $[\mathrm{MD}=-2.3 \mathrm{mg} / \mathrm{dL}(95 \% \mathrm{Cl}-9.5$ to $4.8) ; p=0.523]$. There was no heterogeneity among the reviewed studies.

The trial by Rahbar et al., which used a higher dose of L-carnitine ( $3 \mathrm{~g} /$ day vs. $2 \mathrm{~g} /$ day), found significant increases in triglycerides, apoAI and apoB100 [10]. When this study was not included in the meta-analysis, the aggregated effects of $2 \mathrm{~g} / \mathrm{day}$ of L-carnitine remained not significant $[\mathrm{MD}=-4.4 \mathrm{mg} / \mathrm{dL}(95 \% \mathrm{CI}-13.3$ to
4.6); $\mathrm{p}=0.334$; without associated heterogeneity: $\mathrm{X}^{2}=3.47$, $\mathrm{p}=0.309]$. Santo et al. found that in the carnitine-supplemented group the time for oxidation of LDL particles was significantly prolonged when compared with the placebo group (1.61 vs. $0.91 \mathrm{~h}$ ) [11]. On the other hand, in the study by Malaguarnera et al. the concentration of oxidised-LDL particles was significantly lower in patients who received L-carnitine (43.1 vs. $55.0 \mathrm{U} / \mathrm{L}$, $\mathrm{p}<0.001)[12]$.

\section{Effects on weight}

3 studies evaluated the evolution of weight by means of BMI. After the aggregation of the results, there was no significant difference associated with the administration of L-carnitine $\left[\mathrm{MD}=-0.3 \mathrm{~kg} / \mathrm{m}^{2}(95 \% \mathrm{Cl}-2.4\right.$ to 1.7$) ; \mathrm{p}=0.751$; without heterogeneity: $\left.\mathrm{X}^{2}=1.39, \mathrm{p}=0.499\right)$ ].

\section{Effects on BP}

BP was only evaluated in the study by Santo et al., without finding significant differences at the end of the follow up.

\section{Effects on OS}

2 studies evaluated OS, but they followed different methodologies. In the study by Santo et al., the use of L-carnitine was associated with significant reductions in malondialdehyde, 4-hydroxynonenal and the nitrite/nitrate ratio, while in the study by Malaguarnera et al. L-carnitine administration was associated with lower concentrations of TBARS and conjugated dienes. The data could not be aggregated for meta-analysis.

\section{Discussion}

$\nabla$

Carnitine is a nutrient that plays a key role in the metabolism of FA. It is well known that in the pathogenesis of T2DM disturbances of glucose and lipid metabolism (glucotoxicity and lipotoxicity, respectively) interact, as well as an increase in oxidative stress. Carnitine appears therefore as a potential therapeutic agent for this disease.

This review verifies that few studies with adequate methodological quality to assess the efficacy of carnitine have been published. The results of this meta-analysis support a favourable

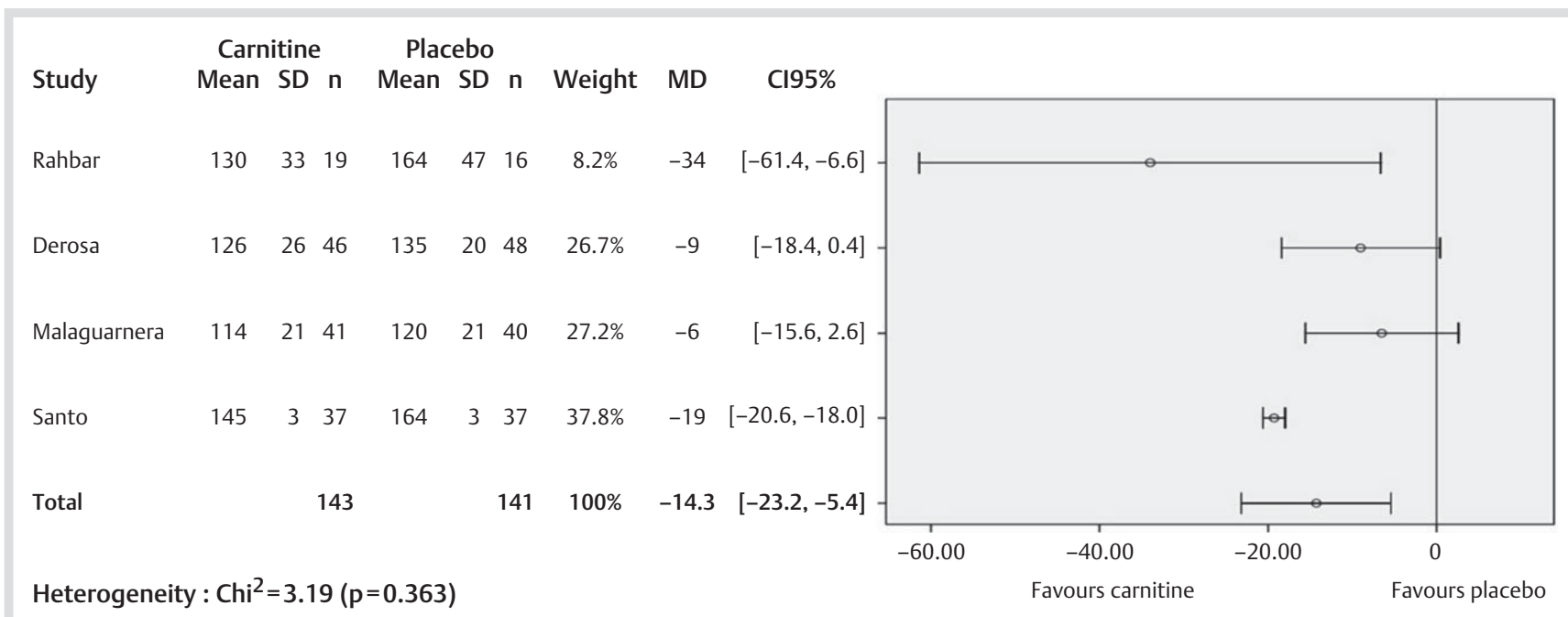

Fig. 2 Forest plot of studies that evaluated the effect of L-carnitine supplementation on fasting plasma glucose (circles represent effect size; extended lines show $95 \%$ confidence intervals). 


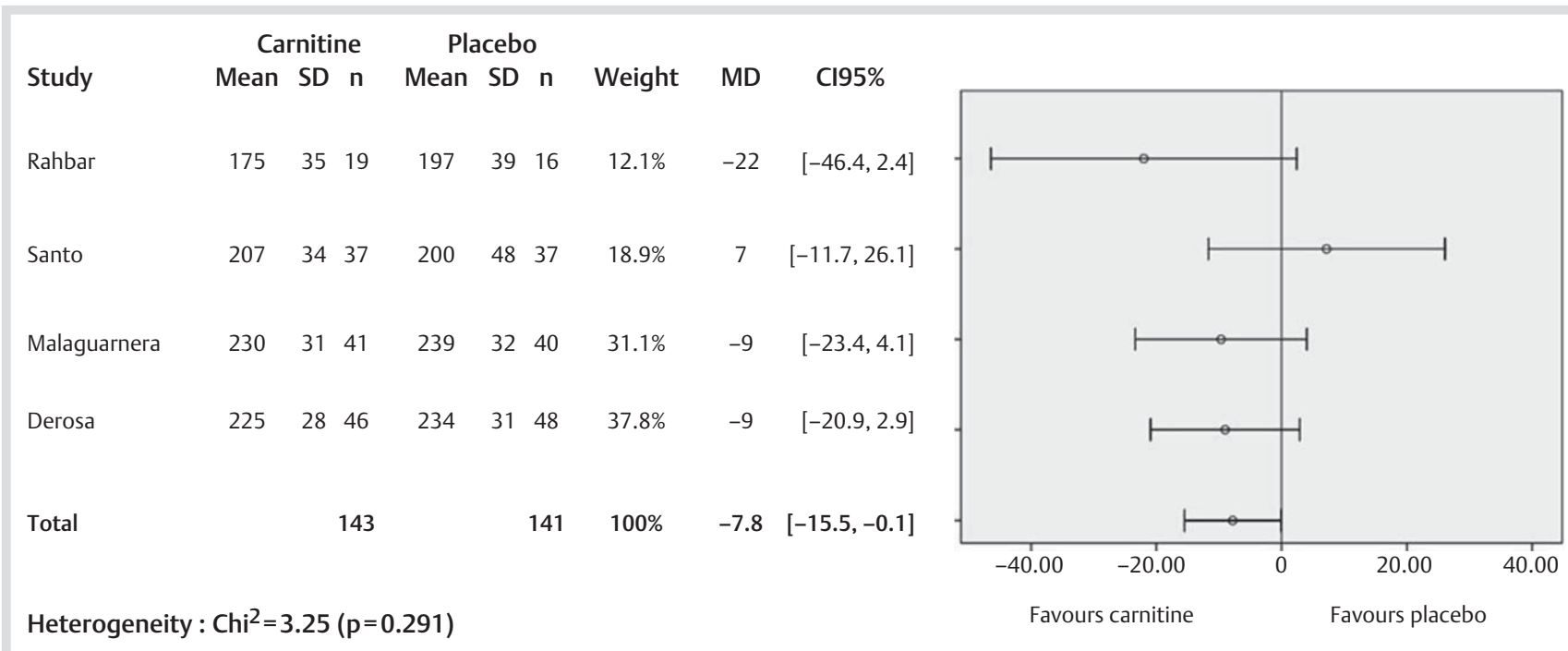

Fig. 3 Forest plot of studies that evaluated the effect of L-carnitine supplementation on total cholesterol (circles represent effect size; extended lines show $95 \%$ confidence intervals).

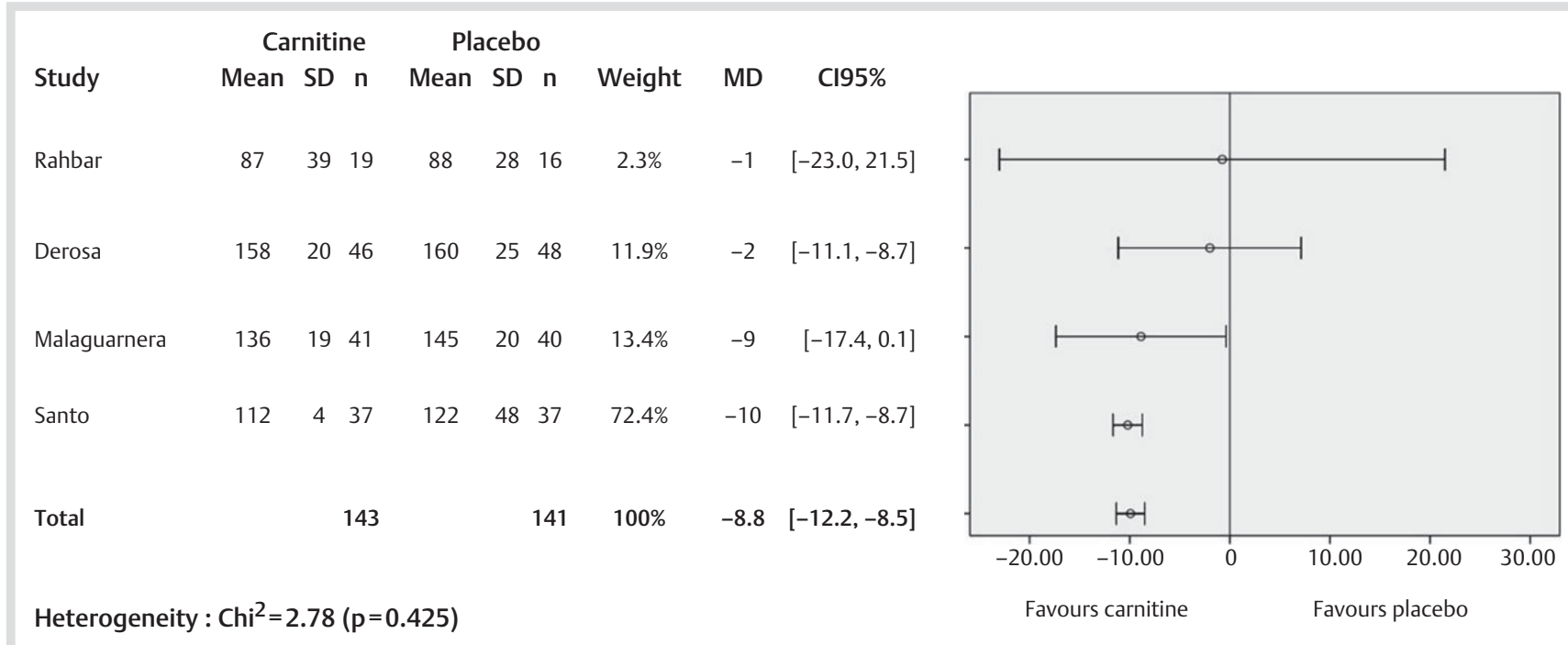

Fig. 4 Forest plot of studies that evaluated the effect of L-carnitine supplementation on LDL-cholesterol (circles represent effect size; extended lines show $95 \%$ confidence intervals).

effect on fasting glucose that did not result in a significant improvement in glycaemic control, as measured by HbA1c. The changes in fasting glucose were moderate, and the study that assessed postprandial glucose did not find significant effects with the administration of $\mathrm{L}$-carnitine, thus it is possible that the improvement of glucose metabolism was insufficient to reach changes in glycated hemoglobin. Some animal and human studies have suggested that carnitine deficiency impairs insulin-sensitivity and can produce elevations of fasting glucose. Carnitine can improve glucose metabolism by means of several mechanisms. First, the enhancement of mitochondrial oxidation of long chain-AcylCoA, which accumulation produces insulinresistance in muscle and heart. Second, inducing changes in glycolitic and gluconeogenic enzymes. Third, modifying the expression of genes related to the insulin signaling cascade. And finally, improving the glucose utilization by heart [14]. These mechanisms were not evaluated in any of the collected studies of this systematic review.
The effects on lipid metabolism focused on reductions in total cholesterol, LDL and B100, all of them atherogenic particles, but the levels of apo AI were also decreased. The last lipoprotein is a constituent of HDL particles, and it is therefore potentially antiatherogenic. On the other hand, triglyceride levels were not affected, a surprising result given the role of L-carnitine in the metabolism of FA. The fact that all lipid analysis were performed whilst in a fasting state, and that in T2DM patients postprandial concentrations of triglycerides and FA may be increased, means that it is possible that the beneficial effects on these parameters have not been properly evaluated [15]. Furthermore, these lipid abnormalities may be related to the phenomena of insulin resistance and lipotoxicity, the mechanisms by which carnitine could improve glucose metabolism. A dose-dependent effect should be considered as well, as beneficial effects could be observed using $2 \mathrm{~g} /$ day of L-carnitine, but doses of $3 \mathrm{~g} /$ day were related to increases in triglycerides concentrations in the study by Rahbar et al. [11]. The results for oxidative stress are incom- 
plete, as is the case with other antioxidants studied in T2DM $[16,17]$. Finally, we did not find heterogeneity among the included studies, although the Q test may underestimate it when few studies are included for meta-analysis.

In conclusion, administration of oral L-carnitine at doses of 2-3 g/day in type 2 Diabetes Mellitus patients is associated with improvements in fasting glucose and decreases in total cholesterol, LDL, and apolipoproteins B100 and AI. Further trials should clarify the effects of L-carnitine on insulin resistance and postprandial lipid dysmetabolism.

Authorship: AVC and LMLP performed the search of articles. All the authors assessed the articles, extracted the data and revised the manuscript. AVC performed the statistical analysis.

Competing interest: Vegenat S.A. (Badajoz, Spain) has provided financial support to this project. The authors have no conflicts of interest to declare.

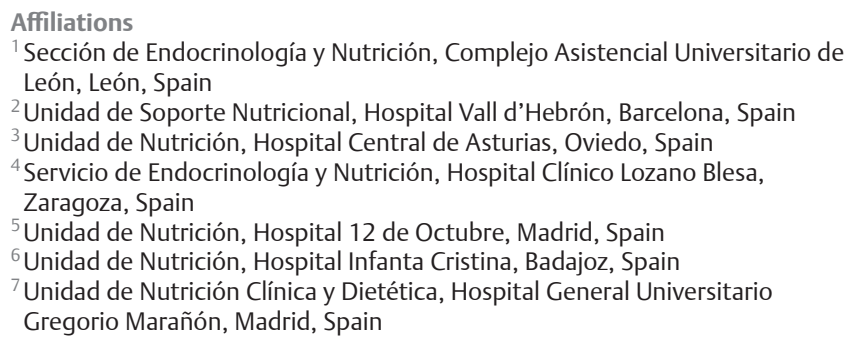

${ }^{1}$ Sección de Endocrinología y Nutrición, Complejo Asistencial Universitario de León, León, Spain

2 Unidad de Soporte Nutricional, Hospital Vall d'Hebrón, Barcelona, Spain

Unidad de Nutrición, Hospital Central de Asturias, Oviedo, Spain

${ }^{4}$ Servicio de Endocrinología y Nutrición, Hospital Clínico Lozano Blesa,

Zaragoza, Spain

${ }^{5}$ Unidad de Nutrición, Hospital 12 de Octubre, Madrid, Spain

${ }^{6}$ Unidad de Nutrición, Hospital Infanta Cristina, Badajoz, Spain

Unidad de Nutrición Clínica y Dietética, Hospital General Universitario

Gregorio Marañón, Madrid, Spain

\section{References}

1 Mingrone G. Carnitine in type 2 diabetes. Ann N Y Acad Sci 2004; 1033: 99-107

2 Mynatt RL. Carnitine and type 2 diabetes. Diabetes Metab Res Rev 2009; 25: S45-S49

3 Ferrannini E, Buzzigoli G, Bevilacqua $S$ et al. Interaction of carnitine with insulin-stimulated glucose metabolism in humans. Am J Physiol 1988; 255: E946-E952
4 Stephens FB, Constantin-Teodosiu D, Greenhaff PL. New insights concerning the role of carnitine in the regulation of fuel metabolism in skeletal muscle. J Physiol 2007; 581: 431-444

5 Stephens FB, Constantin-Teodosiu D, Laithwaite $D$ et al. An acute increase in skeletal muscle. J Clin Endocrinol Metab 2006; 91: 5013-5018

6 Mingrone G, Greco AV, Capristo $E$ et al. L-carnitine improves glucose disposal in type 2 diabetic patients. J Am Coll Nutr 1999; 18: 77-82

7 Capaldo B, Napoli R, Di Bonito P et al. Carnitine improves peripheral glucose disposal in non-insulin-dependent diabetic patients. Diabetes Res Clin Pract 1991; 14: 191-195

8 Poorabbas A, Fallah F, Bagdadchi J et al. Determination of free L-carnitine levels in type II diabetic women with and without complications. Eur J Clin Nutr 2007; 61: 892-895

9 Ringseis R, Keller J, Eder K. Role of carnitine in the regulation of glucose homeostasis and insulin sensitivity: evidence from in vivo and in vitro studies with carnitine supplementation and carnitine deficiency. Eur J Nutr 2012; 51: 1-18

10 Derosa G, Cicero AF, Gaddi A et al. The effect of L-carnitine on plasma lipoprotein(a) levels in hypercholesterolemic patients with type 2 diabetes mellitus. Clin Ther 2003; 25: 1429-1439

11 Rahbar AR, Shakerhosseini R, Saadat $N$ et al. Effect of L-carnitine on plasma glycemic and lipidemic profile in patients with type 2 diabetes mellitus. Eur J Clin Nutr 2005; 59: 592-596

12 Santo SS, Sergio N, Luigi DP et al. Effect of LPC on functional parameters and oxidative profile in type 2 diabetes-associated PAD. Diabetes Res Clin Pract 2006; 72: 231-237

13 Malaguarnera $M$, Vacante $M$, Avitabile $T$ et al. L-carnitine supplementation reduces oxidiced LDL cholesterol in patients with diabetes. Am J Clin Nutr 2009; 89: 71-76

14 Ringseis R, Keller J, Eder J. Role of carnitine in the regulation of glucose homeostasis and insulin sensitivity: evidence from in vivo and in vitro studies. with carnitine supplementation and carnitine deficiency. Eur J Nutr 2012; 51: 1-18

15 Ceriello A, Quagliaro L, Piconi L et al. Effect of postprandial hypertriglyceridemia and hyperglycemia on circulating adhesion molecules and oxidative stress generation and the possible role of simvastatin treatment. Diabetes 2004; 53: 701-710

16 Cuerda C, Luengo LM, Valero MA et al. Antioxidantes y diabetes mellitus: revisión de la evidencia. Nutr Hosp 2011; 26: 68-78

17 Valero MA, Vidal A, Burgos $R$ et al. Meta-analysis on the role of lycopene in type 2 diabetes mellitus. Nutr Hosp 2011; 26: 1236-1241 